\title{
Granzyme B enters the mitochondria in a Sam50-, Tim22- and mtHsp70-dependent manner to induce apoptosis
}

\author{
Valentina Chiusolo ${ }^{1,7}$, Guillaume Jacquemin ${ }^{1,7}$, Esen Yonca Bassoy ${ }^{1}$, Laurent Vinet ${ }^{2,3}$, Lavinia Liguori ${ }^{4}$, Michael Walch ${ }^{5}$, \\ Vera Kozjak-Pavlovic ${ }^{6}$ and Denis Martinvalet ${ }^{\star}, 1$
}

We have found that granzyme $B$ (GB)-induced apoptosis also requires reactive oxygen species resulting from the alteration of mitochondrial complex I. How GB, which does not possess a mitochondrial targeting sequence, enter this organelle is unknown. We show that GB enters the mitochondria independently of the translocase of the outer mitochondrial membrane complex, but requires instead Sam50, the central subunit of the sorting and assembly machinery that integrates outer membrane $\beta$-barrel proteins. Moreover, GB breaches the inner membrane through Tim22, the metabolite carrier translocase pore, in a mitochondrial heat-shock protein 70 (mtHsp70)-dependent manner. Granzyme A (GA) and caspase-3 use a similar route to the mitochondria. Finally, preventing GB from entering the mitochondria either by mutating lysine 243 and arginine 244 or depleting Sam50 renders cells more resistant to GB-mediated reactive oxygen species and cell death. Similarly, Sam50 depletion protects cells from GA-, GM- and caspase-3-mediated cell death. Therefore, cytotoxic molecules enter the mitochondria to induce efficiently cell death through a noncanonical Sam50-, Tim22- and mtHsp70-dependent import pathway.

Cell Death and Differentiation (2017) 24, 747-758; doi:10.1038/cdd.2017.3; published online 24 March 2017

Cytotoxic lymphocytes eradicate pathogen-infected or transformed cells mainly through the cytotoxic granule pathway. ${ }^{1-4}$ Among the five human granzymes $(A, B, H, K$ and $M)$, granzyme $B(G B)$ triggers cell death in both a caspasedependent and caspase-independent manner, although human and mouse GB differ in their requirement for caspase activation. ${ }^{5-10}$ GB-induced cell death also involves Bid/Bax/ Bak-mediated mitochondrial outer membrane permeabilization for the release of the apoptogenic factors cytochrome $c$, Smac/Diablo, Endo G, HtrA2 and AIF., ${ }^{1,-17}$ We have recently reported that mitochondrial reactive oxygen species (ROS) have a critical role in GB pathway by amplifying apoptogenic factor release, oligonucleosomal DNA fragmentation and lysosomal membrane rupture. ${ }^{18}$ Mitochondrial ROS resulted from GB-mediated cleavage of NDUFV1, NDUFS1 and NDUFS2, three subunits of mitochondrial complex I. ${ }^{18}$ How GB that does not possess a mitochondrial targeting sequence enters the mitochondria is not known. The mitochondrial nucleoid encodes only for 13 structural proteins, while the rest of the mitochondrial proteome is nuclear-encoded and transported to the mitochondria through a sophisticated protein import machinery. ${ }^{19-28}$ The translocase of the outer mitochondrial membrane (TOM), the entry gate to the mitochondria, ${ }^{20}$ passes on the cargos to the sorting and assembly machinery (SAM) complex, to the mitochondrial intermembrane space assembly (MIA) complex and to the translocases of the inner mitochondrial membrane TIM22 or TIM23 complexes for delivery to the outer membrane, the intermembrane space, the inner membrane or the matrix, respectively. ${ }^{19-22,25,26,28}$ Unexpectedly, we found that GB mitochondrial entry is independent of the TOM-TIM23 complexes, but requires instead the Sam50 and Tim22 channels and mitochondrial heat-shock protein 70 (mtHsp70). Sam50 depletion protected cells from GB-, GA-, GM- and caspase-3-mediated cell death. Therefore, cytotoxic molecules need to enter the mitochondria to induce cell death.

\section{Results}

GB mitochondrial entry is TOM40-independent. To confirm that GB enters the mitochondria, a fluorescence resonance energy transfer (FRET)-based approach was used. HeLa cells were transfected with a GB-FRET reporter consisting of ECFP connected to EYFP with a linker containing a GB cleavage site VGPDFGR ${ }^{29}$ that was targeted either to the cytosol or to the mitochondrial matrix (GB-cyto-FRET and GB-mito-FRET) (Supplementary Figures $1 \mathrm{~A}$ and $\mathrm{B}$ ). GB and perforin $(P)$ treatment triggered a loss of FRET signal from the mito-FRET reporter with a similar kinetic to that of the cytosolic reporter, indicating that GB entered the mitochondrial matrix (Figures $1 \mathrm{a}-\mathrm{C}$ and Supplementary Figures $1 \mathrm{~A}$ and $\mathrm{B}$ ).

\footnotetext{
${ }^{1}$ Department of Cell Physiology and Metabolism, University of Geneva, Rue Michel-Servet-1, Geneva, Switzerland; ${ }^{2}$ Department of Radiology and Medical Informatics, Geneva University Hospital, Geneva, Switzerland; ${ }^{3}$ Institute of Translational Molecular Imaging, University of Geneva, Geneva, Switzerland; ${ }^{4}$ IBS/Groupe Channels, 71 avenue des Martyrs CS 10090, Grenoble 38044, France; ${ }^{5}$ Anatomy Unit, Department of Medicine, University of Fribourg, Fribourg, Switzerland and ${ }^{6}$ Biozentrum Department of Microbiology, Am Hubland, Würzburg 97074, Germany

*Corresponding author: D Martinvalet, Department of Cell Physiology and Metabolism, University of Geneva, Rue Michel-Servet-1, Geneva 1211, Switzerland. Tel: +41 22379 5285; Fax: +41 2237952 60; E-mail: Denis.Martinvalet@unige.ch

${ }^{7}$ These authors contributed equally to this work.

Received 31.10.16; revised 07.12.16; accepted 23.12.16; Edited by L Scorrano; published online 24.3.2017
} 
Moreover, native and recombinant GB entered the purified intact mouse liver and yeast mitochondria in a valinomycinsensitive manner, as the membrane potential is required for this import (Supplementary Figures $1 \mathrm{C}-\mathrm{E}$ ). This import was independent of GB catalytic activity, as inactive S-AGB also entered the mitochondria from both species. Moreover, gentle a

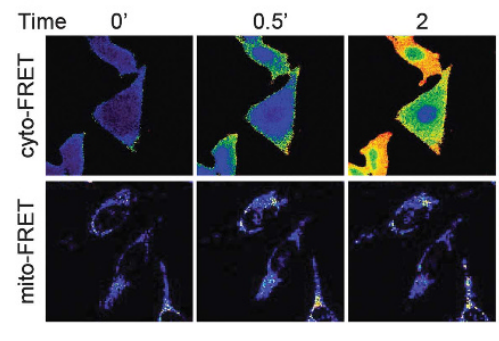

d

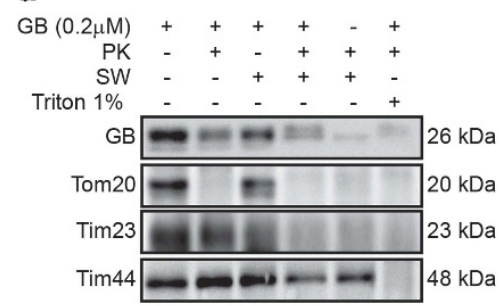

h

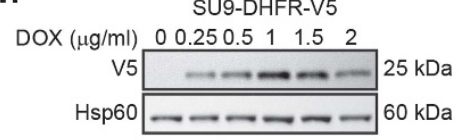

i

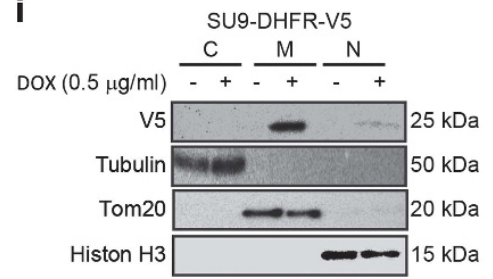

I

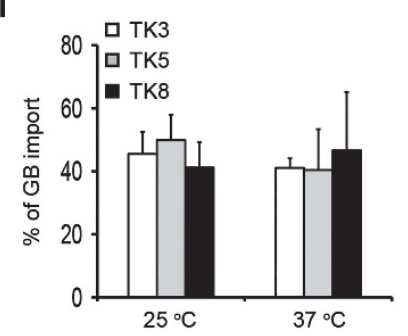

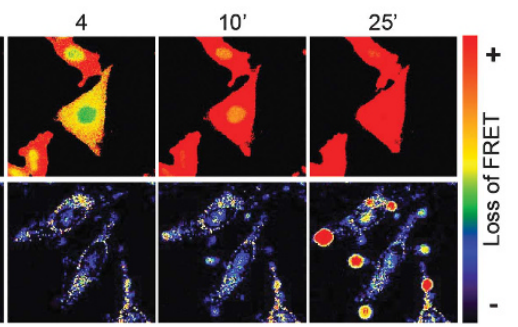

e

$\operatorname{DOX}(\mu \mathrm{g} / \mathrm{ml}) \quad 0 \quad 0.250 .5 \quad 1 \quad 1.5 \quad 2$

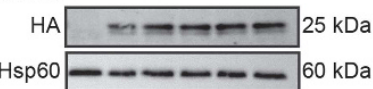

f

f $\operatorname{DOx}(0.5 \mu \mathrm{g} / \mathrm{ml}) \frac{\mathrm{C}}{-}+\frac{\mathrm{M}}{-+} \frac{\mathrm{N}}{-+}$

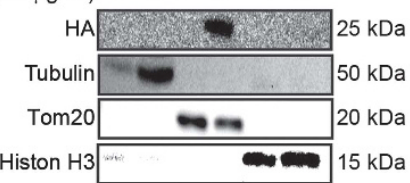

j

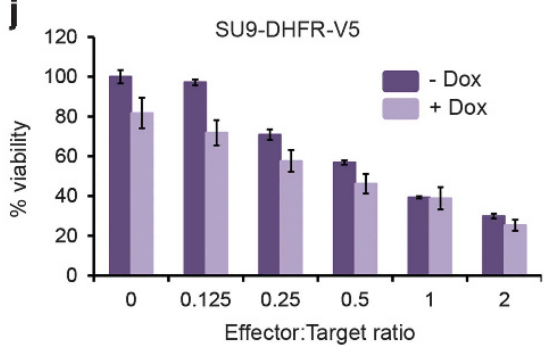

$\mathbf{m}_{\text {SU9-DHFR }}++++++$

$\begin{array}{ccccccc}\text { Valino } & - & - & + & - & - & + \\ T\left({ }^{\circ} \mathrm{C}\right) & 25 & 37 & 25 & 25 & 37 & 25\end{array}$

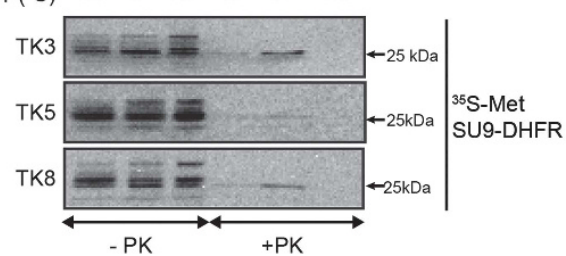

b

C

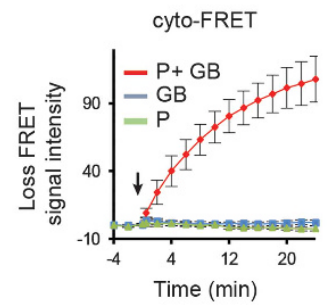

g

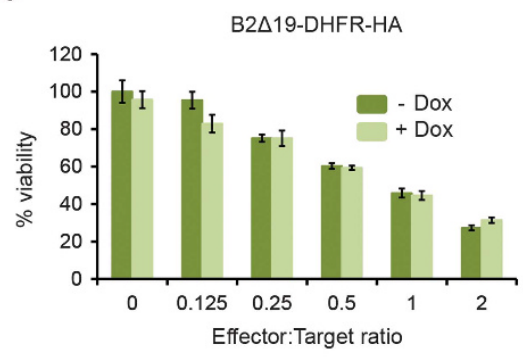

k

$(0.2 \mu \mathrm{M})-++++++$

$\begin{array}{ccccccccc}\text { Valino } & - & - & - & + & - & - & - & + \\ \mathrm{T}\left({ }^{\circ} \mathrm{C}\right) & 25 & 25 & 37 & 25 & 25 & 25 & 37 & 25\end{array}$

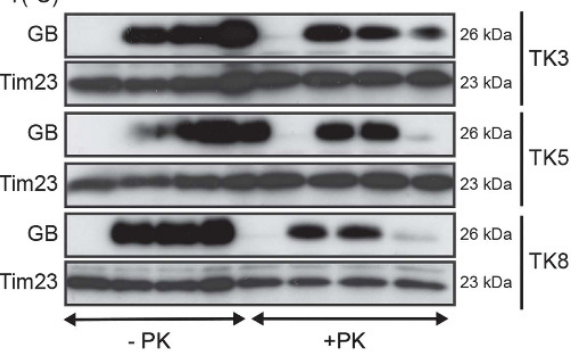

Figure 1 GB enters the mitochondria in a TOM40-independent manner. (a) GB enters the HeLa cell mitochondria. HeLa cells overexpressing cytosolic (top row) or mitochondrial (bottom row) GB-FRET reporters were treated with perforin plus GB. GB activity was monitored by the loss of the FRET signal using confocal microscopy. Images are representative of three independent experiments. Quantification of the loss of FRET signal for cyto-FRET (b) and mito-FRET (c), mean \pm S.D. of at least 35 cells in three independent experiments. (d) Freshly purified yeast mitochondria were incubated or not with GB in import buffer before gentle treatment with proteinase K (PK) in the absence or presence of a swelling step or direct solubilization with $1 \%$ Triton X-100. (e) HeLa cell expression of inducible HA-tagged B2 19 -DHFR following $96 \mathrm{~h}$ DOX treatment. (f) Cells treated as in (e) were fractionated into cytosolic (C), mitochondrial (M) and nuclear (N) fractions. B2 $\Delta$ 19-DHFR-HA accumulates in the mitochondria. (g) Cells pretreated or not with DOX as in (e) were used as target cells for YT-Indy killer cells, mean \pm S.D. of three independent experiments. (h) Same as in (e) using HeLa cells expressed inducible V5tagged SU9-DHFR. (i) Same as in (f) using HeLa expressing inducible SU9-DHFR-V5. (j) Same as in (g) using HeLa expressing inducible SU9-DHFR-V5, mean \pm S.D. of three independent experiments. (k) Mitochondria purified from yeast carrying wild-type (TK8) or two thermosensitive alleles (TK3 and TK5) of Tom40 were used in the import assay of $\mathrm{GB}$ at $25^{\circ} \mathrm{C}$ or $37^{\circ} \mathrm{C}$ permissive and non-permissive temperature, respectively. Western blots are all representative of three independent experiments. (I) Same as in (k), mean \pm S.D. of three independent experiments. $(\mathbf{m})$ Same as in (k) using ${ }^{35}$ S-Met-labeled SU9-DHFR ( ${ }^{35}$ S-Met SU9), a positive control known to enter the mitochondria through Tom40 pathway. Autoradiogram is representative of three independent experiments 
proteinase K pretreatment inhibited GB mitochondrial import, indicating that it is a facilitated process (Supplementary Figures 1D and E). Within $1 \mathrm{~min}$, GB enters the mitochondria and reaches a plateau by $10 \mathrm{~min}$, indicating that this import is fast and specific (Supplementary Figure 1F). Freshly purified yeast mitochondria were incubated with or without GB before gentle treatment with proteinase $\mathrm{K}$ in the absence or presence of a swelling step to disrupt the mitochondrial outer membrane to analyze its submitochondrial localization. ${ }^{30,31}$ Similarly to the mitochondrial matrix marker Tim44, some of the $\mathrm{GB}$ is protected from proteolysis even after disruption of the outer mitochondrial membrane, indicating that it is in the matrix (Figure 1d), in agreement with GB ability to cleave the matrix subunits of complex I and the mito-FRET reporter (Figure 1c).

Although GB does not have a mitochondrial targeting sequence, we still tested whether TOM complex was involved. Saturation of mouse liver mitochondria TOM and TIM23 complexes with cold SU9-DHFR (dihydrofolate reductase) and B2 19 -DHFR inhibited in a dose-dependent manner the import of ${ }^{35}$ S-Met-labeled SU9-DHFR ${ }^{23,32-35}$ used throughout this work as a positive control (Supplementary Figure 2A). This inhibition was even more pronounced when cold SU9-DHFR and B2 19 -DHFR were stabilized with methotrexate (MTX) ${ }^{23}$ (Supplementary Figure 2B). However, SU9-DHFR did not inhibit GB import, while B2 $\Delta 19$ did so to a minor level at the highest dose (Supplementary Figure 2C); however, MTX stabilization completely prevented B2 19 -DHFR inhibition of GB import (Supplementary Figure 2D). To further test the effect of B2 19 -DHFR and SU9-DHFR on GB-induced cell death, HeLa cells expressing HA-tagged B2 $\Delta 19$-DHFR upon DOX treatment (Figure 1e) were fractionated into cytosolic $(C)$, mitochondrial $(\mathrm{M})$ and nuclear $(\mathrm{N})$ fractions (Figure 1f). Although B2 $\Delta 19-\mathrm{DHFR}-\mathrm{HA}$ accumulated in the mitochondria and most likely jammed its import machinery, it did not inhibit GB-expressing YT-Indy killing (Figure 1g). Similar results were obtained from HeLa overexpressing SU9-DHFR-V5 (Figures 1h-j).

Since GB entered the yeast mitochondria, we used a genetic approach to dissect this import pathway. Mitochondria purified from yeast strains expressing thermosensitive alleles of Tom40 with increasing severity (TK3 and TK5), or wild-type (wt) allele (TK8), ${ }^{33}$ were used to import GB. GB was efficiently imported in mitochondria from all strains regardless of the temperature (Figures $1 \mathrm{k}$ and I), whereas, as reported, mitochondria from TK5 did not import ${ }^{35} \mathrm{~S}-M e t-$ SU9-DHFR $^{33}$ (Figure $1 \mathrm{~m}$ ). Moreover, mitochondria from yeast strains deficient for the receptors Tom70, ${ }^{36}$ Tom20 32,36 or Tom22, the latter in a Tom70 overexpression background $\left(\triangle T\right.$ Tom22),${ }^{37}$ although unable to import properly ${ }^{35} \mathrm{~S}-M e t-S U 9-$ DHFR (Supplementary Figures $2 \mathrm{E}$ and $\mathrm{F}$ ), were still efficiently importing GB (Supplementary Figures 2G and $H$ ). Taken together, these data further support that the TOM complex is not directly involved in GB mitochondrial entry.

GB mitochondrial entry requires Tob55/Sam50. Mature GB N-terminal end is not accessible, whereas its C-terminal tail extends from the globular core of the protein, ${ }^{38}$ suggesting that this $\mathrm{C}$-terminal tail could have a role in its import. To look for granzyme mitochondrial-binding partners, we fused two $B$ domains of protein $A$ to the $N$ terminus of $\mathrm{GA}$ or $\mathrm{GB}$ C-terminal tail, residues Ser231-Val246 and Val231-Tyr245, respectively, or at the C-terminus of full-length GB used as negative control, and performed affinity purification and mass spectrometry analysis (Supplementary Figure $3 \mathrm{~A}$ ). The three voltage-dependent anion channels (VDAC) 1/2/3 isoforms and the coiled-coil helix coiled-coil helix domain-containing protein 3 (CHCHD3), also known as Mic19, were identified as putative interacting partners for the GA and GB C-terminal tail. Mitochondria from yeast strain M22-2-1 deficient for porin 1 and 2, the two yeast orthologs of VDAC, ${ }^{39}$ were unable to import efficiently GB (Supplementary Figures 3B and $\mathrm{C}$ ) as well as ${ }^{35}$ S-Met SU9-DHFR (Supplementary Figure 3D), because of their reduced membrane potential (Supplementary Figure 3E). Similar results were obtained from mouse liver mitochondria pretreated with S-18, a peptide inhibitor of VDAC $^{40}$ (Supplementary Figures 3F and I). Altogether these results exclude VDAC as a putative channel for GB mitochondrial import in agreement with its $3 \mathrm{~nm}$ exclusion diameter being too small to accommodate the $3.5 \times 5 \mathrm{~nm}$ GB ellipsoid. ${ }^{38,41,42}$

Since CHCHD3/Mic19, a member of the mitochondrial contact site and cristae organizing system, ${ }^{43,44}$ also interacts with Sam50 channel, we tested whether this outer membrane channel could be used by GB to enter the mitochondria. From yeast expressing Tob55/Sam50 under the control of the gal promoter grown in galactose or glucose, we obtained mitochondria-overexpressing or -deficient for Tob55/Sam50, while Tom40 and Por1 expression was unaffected ${ }^{45}$ (Supplementary Figure 4A). Loss of Tob55/Sam50 had no effect on ${ }^{35}$ S-Met SU9-DHFR import (Figure $2 \mathrm{~b}$ and Supplementary Figure 4B), whereas it severely compromised GB import (Supplementary Figure 4C). This was further confirmed by repeating the same experiments including mitochondria purified from the parental strain YPH499 grown as described previously (Figures 2a-d and Supplementary Figure 4C). Taken together, these results show that Tob55/ Sam50 is necessary for GB mitochondrial entry. Blue-Native (BN) gel electrophoresis and western blot analysis of mitochondria incubated with or without biotinylated GB for $1 \mathrm{~min}$ showed that GB migrated with an apparent molecular weight of $240 \mathrm{kDa}$, which coincides with TOB55/ SAM50 complex (Figure $2 \mathrm{e}$, left and middle panels). This indicates that GB most likely shuttles through Tob55/Sam50. After $15 \mathrm{~min}, \mathrm{~GB}$ migrated at a higher molecular weight around $300 \mathrm{kDa}$ but lower than the migration of Tom40 complex (Figure 2e, left and right panels). Moreover, Lactococcus lactis, expressing either yeast Por1, Tom40 or Tob55/Sam50 (Supplementary Figures 4D-F), were used as surrogate mitochondria in import assays after lysozyme removal of the peptidoglycan cell wall to prevent nonspecific binding of the cationic GB (Supplementary Figure 4G). Interestingly, only L. lactis expressing Tob55/Sam50 was capable of importing GB (Figures $2 \mathrm{f}-\mathrm{h}$ and Supplementary Figures $4 \mathrm{H}$ and I). Since our GB preparation is completely devoid of any trace of granulysin contamination when tested by mass spectrometry, we can safely conclude that Tob55/ Sam50 is sufficient for GB import in agreement with mitochondria from Tob37-deficient $(\Delta$ Tob37) yeast having no 
a

a

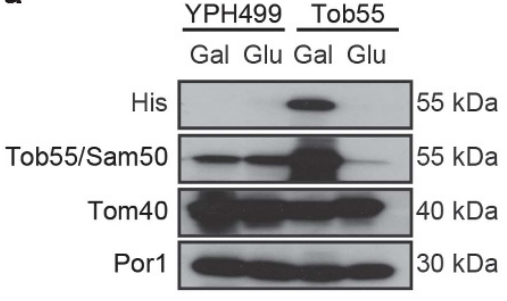

c

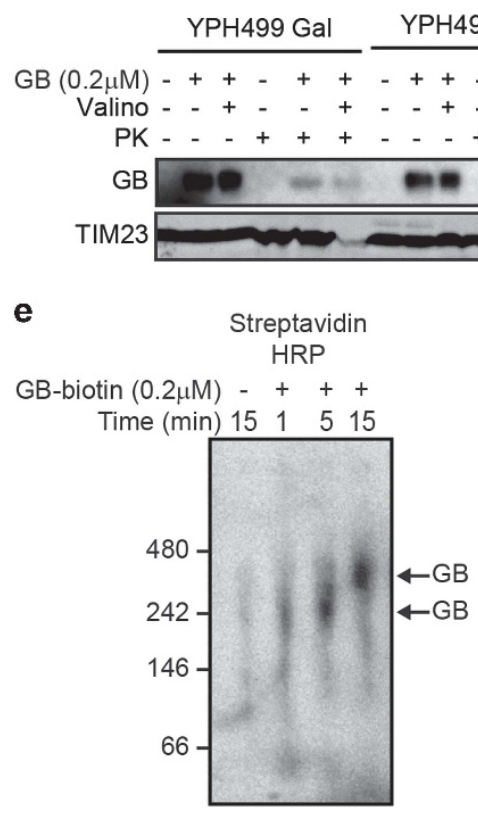

f

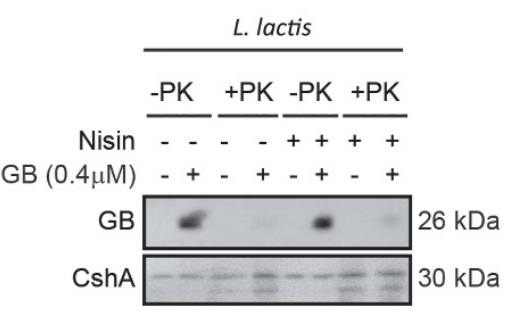

e

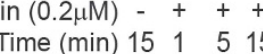

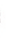

b

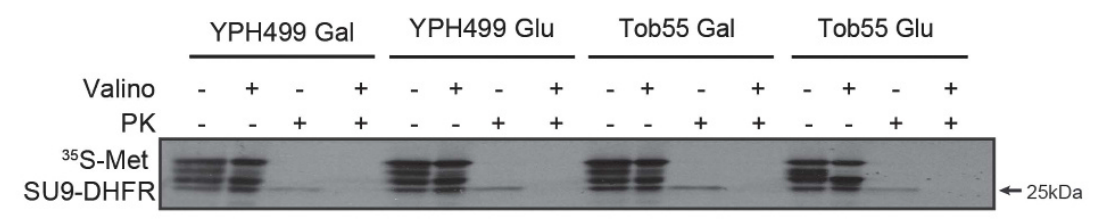

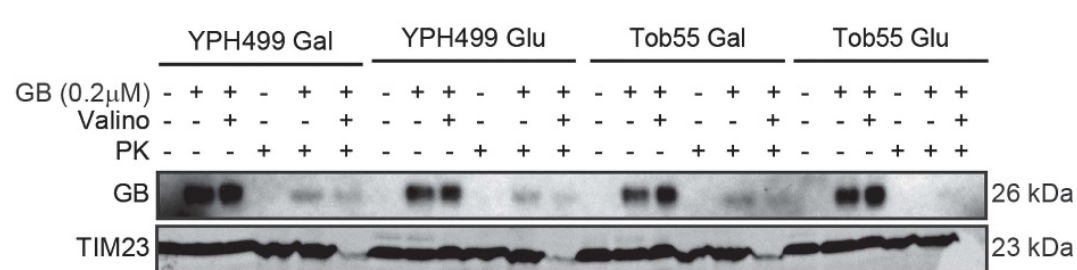

g
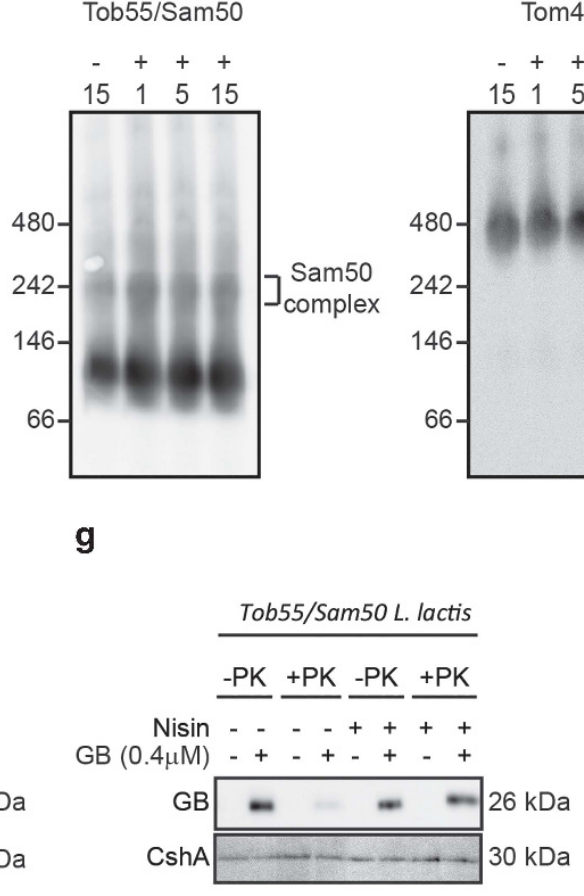

d Buffer

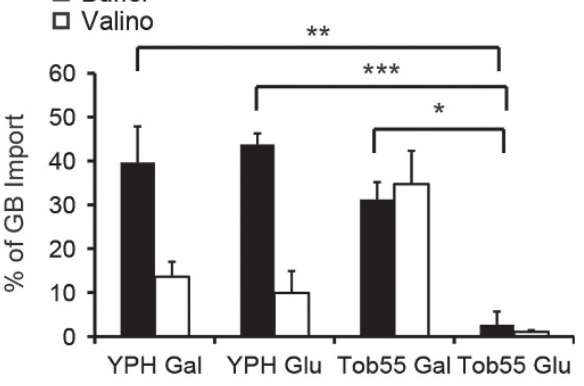

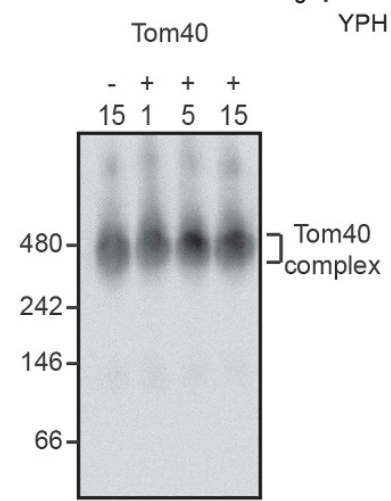

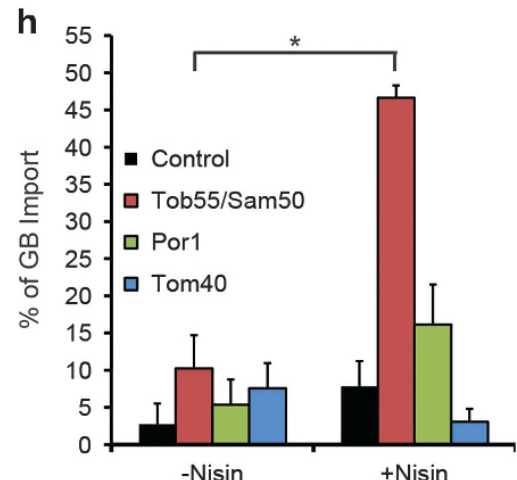

Figure 2 GB mitochondrial entry requires Tob55/Sam50. (a) Mitochondria purified from YPH499 parental yeast strain or a strain expressing Tob55-His under the control of the promoter of the galactose operon (Tob55) grown in galactose or glucose were analyzed for the expression of Tob55/Sam50, Tom40 and Por1 by western blot. Blot is representative of three independent experiments. (b) Mitochondria purified as in (a) were used to import ${ }^{35} \mathrm{~S}$-Met SU9. The autoradiogram is representative of three independent experiments. (c) Same as in (b) for GB import. Blot is representative of four independent experiments. (d) Same as in (c), mean \pm S.D. of four independent experiments. (e) Mitochondria purified from Tob55 yeast strain grown in galactose medium were used in import assay for biotinylated GB (GB-biotin). Samples were resolved by Blue-Native gel electrophoresis and analyzed by western blot with streptavidin-HRP, anti-yeast Tob55 or anti-yeast Tom40. Parental $L$. lactis (f) or $L$. lactis overexpressing yeast Tob55/Sam50 after nisin induction $(\mathbf{g})$ were used in the import assay. Only L. lactis expressing Tob55/Sam50 can import GB. Blots are representative of three independent experiments. (h) Same as in (f-g), mean \pm S.E.M. of three independent experiments. ${ }^{*} P \leq 0.05$, ${ }^{* \star} P \leq 0.01$ and ${ }^{* \star *} P \leq 0.001$ (two-sided $t$-test (d) or Kruskal-Wallis test (h))

defect in importing GB (Supplementary Figure 4J). This is not surprising, as Tob37 promotes the lateral release of $\beta$-barrel proteins from the Tob55/Sam50 channel to the lipid bilayer. ${ }^{46}$ Taken together, these results show that Tob55/Sam50 is required for GB mitochondrial import.
GB residues Lys and Arg 243/244 are required for its mitochondrial import. As stated earlier, GB N-terminal end is not accessible, whereas its C-terminal end is. ${ }^{47}$ In agreement with this observation, only GB C-terminal portion (Val231-Tyr245) but not the N-terminal (Ile16-Tyr29) portion 
triggered the mitochondrial import of DHFR (Figures 3a and b). On the GB C-terminal tail, substitution of His236 and Lys 239-240, part of a cationic residues motif important for GB cytosolic delivery, ${ }^{48}$ did not inhibit the mitochondrial import of recombinant GB, whereas substitution of Lys and Arg 243/244 with Ala did inhibit the mitochondrial import of recombinant $\mathrm{GB}$ (Figures $3 \mathrm{c}$ and $\mathrm{d}$ and Supplementary Figure 5A). ZsGreen fused at the $N$ terminus of wtGB perfectly colocalized with the mitochondria when expressed in HeLa cells (Figures $3 e$ and $f$ ). We obtained similar results for the other four human granzymes (Supplementary Figure 5B). However, Zs-GB KR243/244AA did not colocalize with the mitochondria (Figures $3 e$ and $f$ and Supplementary Figure 5B). The radical substitution of Lys and Arg with the acidic residue Glu did not prevent the mitochondrial localization, suggesting that hydrophilicity and polarity, but not the nature of the charge itself, are important (Supplementary Figure 5B). These results indicate that residues Lys and Arg 243/244 are required for GB mitochondrial import.

GB mitochondrial import is required for cell death. Purified GB KR243/244AA had similar activity as the wt enzyme (Figures $3 \mathrm{~g}$ and $\mathrm{h}$ ). YT-Indy NK cells were silenced for endogenously expressed GB, GK and GM (YT-shGzm) before re-expression of either wtGB (YT-wtGB) or GB KR243/244AA (YT-KR/AA GB). Both YT-wtGB and YT-KR/ AA GB expressed similar levels of GB (Figure 3i) and were able to deliver $\mathrm{GB}$ into target cells in a comparable manner (Figure 3j). Furthermore, YT-wtGB and YT-KR/AA triggered similar PARP-1 and Bid cleavage when delivered into the target cells (Figure 3k). Both type of effector cells also triggered the loss of FRET from GB-cyto-FRET reporter (Figure $3 \mathrm{l}$ and Supplementary Figure $6 \mathrm{~A}$ ), together indicating that both wtGB and $\mathrm{GB} \mathrm{KR} / \mathrm{AA}$ have very similar catalytic activity and are similarly delivered into the target cell cytosol during killer cell attack. However, contrary to YT-wtGB, YT-KR/AA GB poorly triggered the loss of GB-mito-FRET signal as $G B \mathrm{KR} / \mathrm{AA}$ cannot enter the mitochondria (Figure $3 \mathrm{~m}$ and Supplementary Figure 6B). Consequently, YT-KR/AA GB were significantly impaired in their ability to trigger ROS increase in target cells (Figure 3n). YT-wtGB killed HeLa-CD80 cells like the parental YT-Indy, whereas YT-Indy re-expressing GB KR/AA killed less efficiently HeLaCD80 target cells in a 4-h assay (Figure 3o). During a longer time period, YT-KR/AA GB killed more than YT-shGzm expressing only perforin, although significantly less efficiently than YT-wtGB (Supplementary Figure 6C). Adoptive transfer of YT-shGB or YT-KR/AA GB was not able to reduce significantly the tumor burden of tumor-bearing NOD/SCID mice compared with the control animals (Figures $3 p$ and $q$ ). However, the tumor burden was significantly reduced in animals receiving YT-wtGB (Figures $3 p$ and $q$ ). Taken together, these results clearly indicate that GB entry in target cell mitochondria is critical for ROS production and fast and efficient cell death induction during both in vitro and in vivo killer cell attack.

HeLa cells with inducible shRNA against Tob55/Sam50 or Tom $40^{49}$ (Figures $4 a$ and b) were induced with DOX to obtain partial Tob55/Sam50 or Tom40 knockdown as they are essential proteins, ${ }^{50}$ and used as target for parental YT-Indyexpressing GB. Three days of DOX induction was sufficient for the loss of Tob55/Sam50 without altering the expression of Tom40 and vice versa (Figures $4 \mathrm{a}$ and $\mathrm{b}$ ). The loss of Tob55/ Sam50, but not that of Tom40, protected target cells against GB-expressing effector cells (Figures 4c and d), whereas both types of target cells were still sensitive to UV or the anti-Fas agonist antibody (Supplementary Figures 7A and B). Moreover, cells knocked down for Tob55/Sam50 still experienced Bid and PARP-1 cleavage (Figure $4 e$ ) when treated with GB and $P$; however, the release of cytochrome $c$ was reduced in Tob55/Sam50-depleted cells (Figure 4f), indicating that GB mitochondrial entry is needed for apoptogenic factor release, most likely following the production of ROS. ${ }^{18,51}$

GA, GM and caspase-3 induce cell death in a Tob55/ Sam50-dependent manner. Similar to GB, GA localizes in the mitochondrial matrix compartment (Figure 5a), enters the yeast mitochondria independently of Tom40 (Figure 5b) and its import was neither affected by saturation of the canonical mitochondrial import machinery with B2 $\Delta 19-\mathrm{DHFR}$ in the presence of MTX (Supplementary Figures $8 \mathrm{~A}$ and $\mathrm{B}$ ) nor by the loss of Tom70, Tom22 and Tom20 (Supplementary Figures $8 \mathrm{C}$ and D). Moreover, yeast mitochondria deficient for Tob55/Sam50 were also unable to import GA (Figure 5c). Like for GB, within 1 min biotinylated GA migrates at around $240 \mathrm{kDa}$ like Tob55/Sam50 complex (Figure 5d, left and middle panels) and by 5 to $15 \mathrm{~min}$ accumulates in larger complexes of about $300 \mathrm{kDa}$ (Figure $5 \mathrm{~d}$, left and right panels). Loss of Sam50, but not loss of Tom40, also protected target cells against YT-Indy expressing only GA (Figures $5 e-g)$. We obtained similar results with YT-Indy expressing only GM (Figures $5 \mathrm{~h}-\mathrm{j}$ ), suggesting that GA and GM requires Tob55/ Sam50 to induce target cell death. Rev-caspase-3 construct encodes an active recombinant caspase- 3 in which the positioning of the small subunit upstream of the large one allows constitutive autocatalytic processing. ${ }^{52}$ Active caspase-3 mitochondrial entry was also found Tom40independent (Figures $6 \mathrm{a}$ and b), and like $\mathrm{GB}$, requires Tob55/Sam50 (Figure 6c). Silencing of Tob55/Sam50 partially but significantly protected from Rev-caspase-3-induced cell death (Figure 6d). This result indicates that Tob55/Sam50 is necessary for active caspase-3 to enter the mitochondria for cell death induction in agreement with the fact that caspase-3 cleaves NDUFS1 to trigger ROS-dependent death. ${ }^{53}$

GA and GB cross the inner mitochondrial membrane in a Tim22- and mtHsp70-dependent manner. Finally, we used the same yeast genetic approach to investigate how GB crosses the inner mitochondrial membrane. Yeast mitochondria purified from the parental strain YPH499 or from YPH Tim23-76ts that carry a thermosensitive allele of Tim23 unable to shuttle cargos into the mitochondrial matrix ${ }^{54}$ were as efficiently able to import both GB (Figures $7 \mathrm{a}$ and b) and GA (Figures 7c and d). Unexpectedly, mitochondria from YPH-Tim22-14ts strain that carry a thermosensitive allele of Tim22 incapable of the membrane insertion of the cargos $^{55}$ were significantly reduced in their ability to import both GB (Figures $7 a$ and b) and GA (Figures 7c and d). 
Although surprising, these results are in agreement with the later time points BN-gel migration pattern of biotinylated granzymes of around $300 \mathrm{kDa}$, which coincide with the size of
TIM22 complex (Figures 2e and 5c). Moreover, we also found that mitochondria purified from the yeast strain carrying the thermosensitive allele of mitochondrial Hsp70 (mtHsp70) a

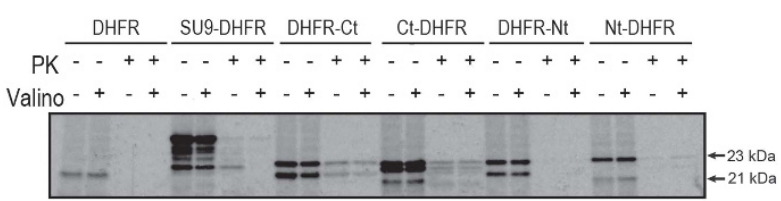

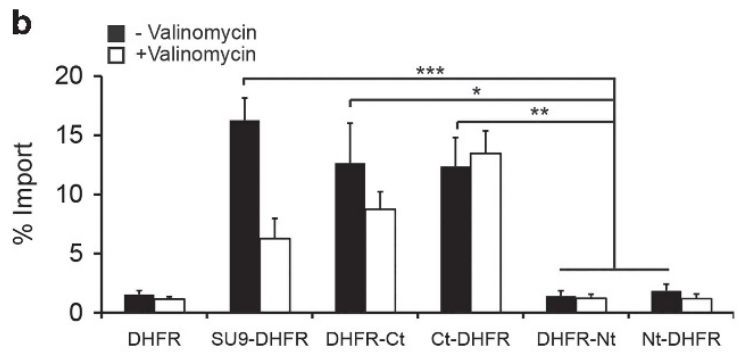

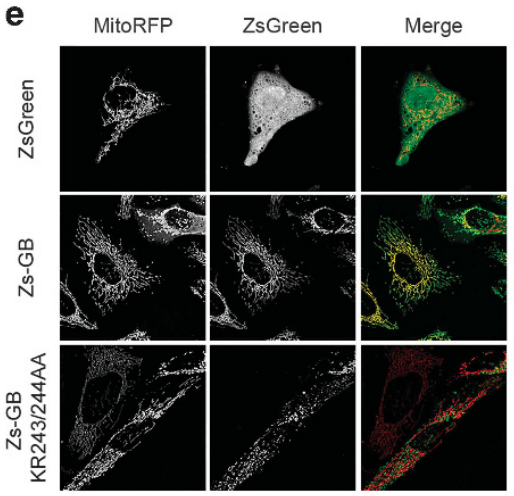

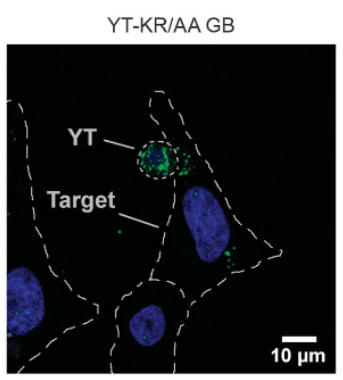

$\mathbf{k}$

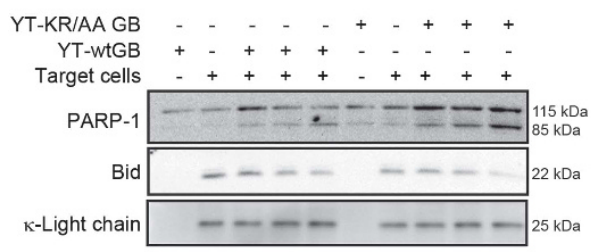

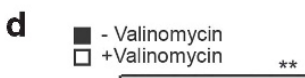

$\mathrm{PK} \frac{\mathrm{B}}{-+} \frac{\mathrm{GB}}{+++\ldots++} \frac{\mathrm{HKK}-\mathrm{AAA}}{\ldots++}$

Valino $-+\ldots++\ldots+\ldots+\ldots+$

$\mathrm{GB} \longrightarrow--\infty{ }_{26 \mathrm{kDa}}$

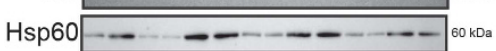
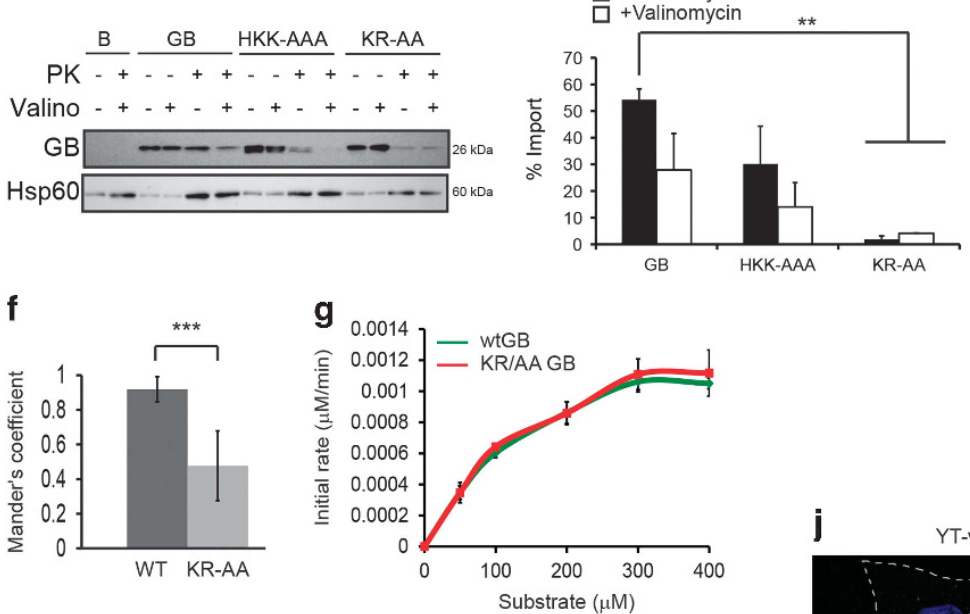

h

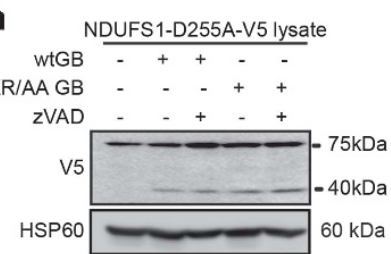

i
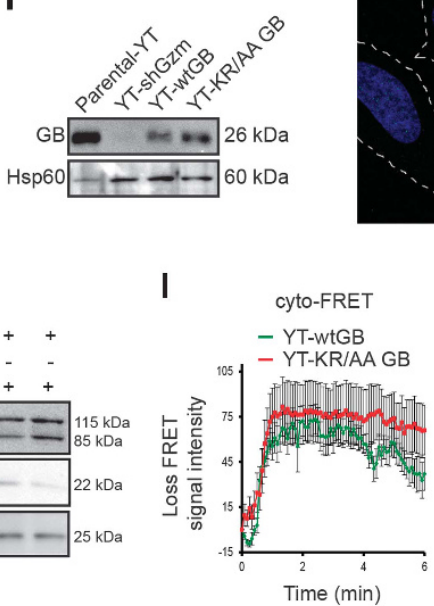

m

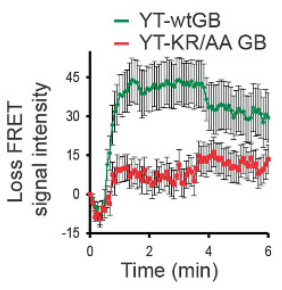

n

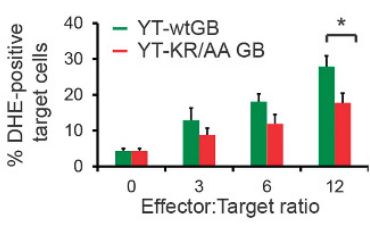

$\mathbf{0}$

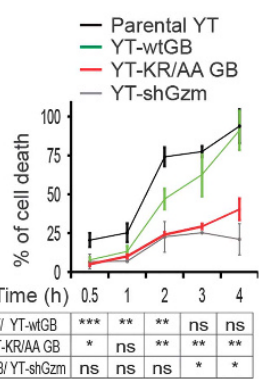

p

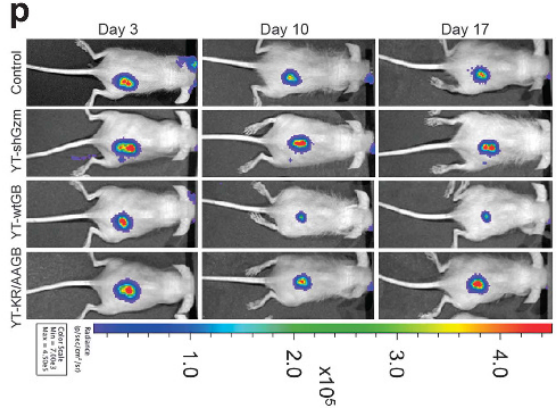

$\mathbf{q}$

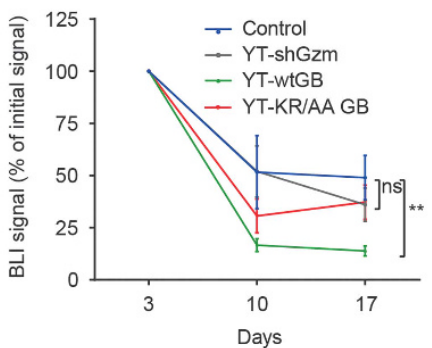


a

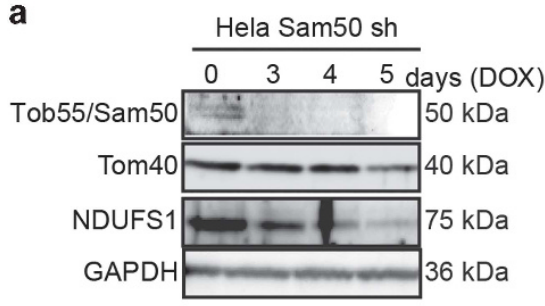

c

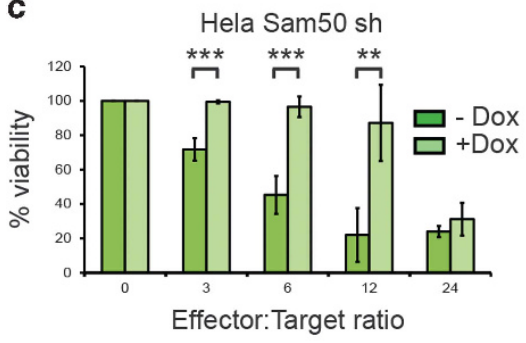

e

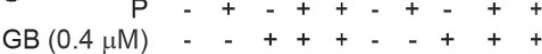
Time (min) $6060603060606060 \quad 3060$

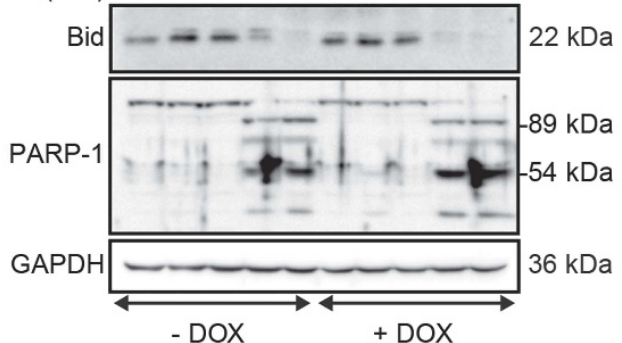

d b

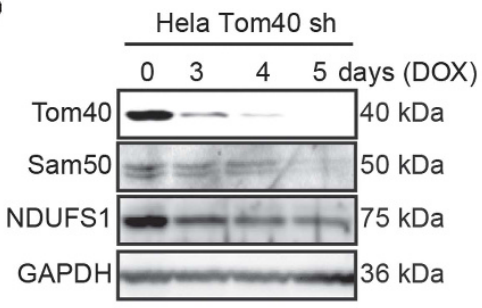

Hela Tom40 sh

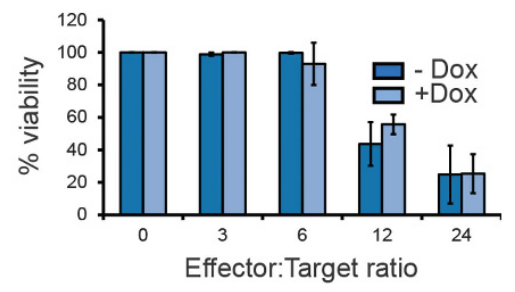

f

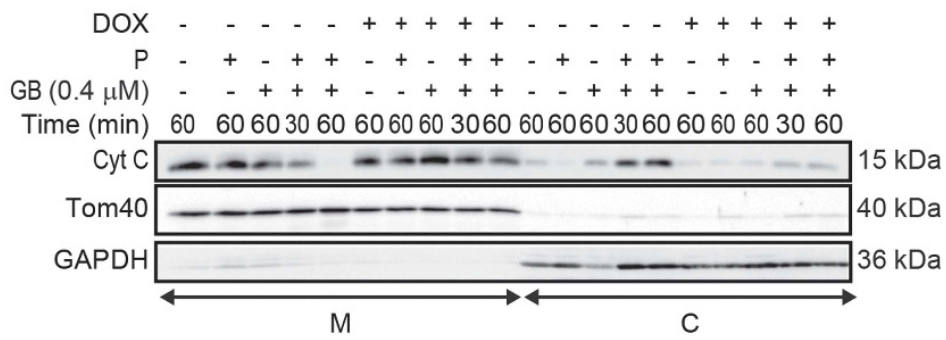

Figure 4 Tob55/Sam50-deficient cells are resistant to GB-induced cell death. Tob55/Sam50, Tom40, NDUFS1 and GAPDH protein level in HeLa cells expressing a DOX-inducible short hairpin RNA (shRNA) against Sam50 (a) or Tom40 (b) in the absence or presence of DOX induction. Hela cells were silenced for Tob55/Sam50 (c) or Tom40 (d) following 3 days of DOX treatment and used as targets for parental YT-Indy. (e) Cells silenced or not for Tob55/Sam50 for 4 days were treated with a sublytic dose of P plus GB before Bid and PARP-1 cleavage analysis by western blot. (f) Cells treated as in (e) before fractionation into cytosolic $(\mathrm{C})$ and mitochondrial (M) fractions for the analysis of cytochrome $c$ release by western blot. Blots are all representative of three independent experiments. Data in (c) and (d) are mean \pm S.D. of three independent experiments. ${ }^{\star \star} P \leq 0.01,{ }^{* \star} P \leq 0.001$ (one-sided $t$-test)

Figure 3 GB mitochondrial entry is required for cell death. (a) The GB C terminus (V231-Y245) or N terminus (I21-Y34) were fused either in N or C terminus of DHFR that were ${ }^{35} \mathrm{~S}$-labeled and used in import assay. Autoradiogram is representative of five independent experiments. (b) Quantification of (a) mean \pm S.D. of five independent experiments. (c) Recombinant wild-type (wt) GB or GB carrying the triple mutation H236A KK239/240AA or the double mutation KR243/244AA were used in import assay with mouse liver mitochondria. For the imported fraction (+PK), three time more sample was loaded. Western blot is representative of three independent experiments. (d) Quantification of (c), mean \pm S.D. of two independent experiments. (e) HeLa cells overexpressing mitoRFP and ZsGreen fused to the N-terminus of wtGB (Zs-GB) or KR243/244AA mutant GB (Zs-GB KR243/244AA) were imaged by confocal microscopy. Images are representative of three independent experiments. (f) Same as in (e), colocalization index analyzed by Mander's coefficient; $n=45$ cells (Zs-GB) and $n=42$ cells (Zs-GB KR243/244AA). (g) Purified wtGB or KR243/244AA GB showed similar specific activity when tested against a GB colorimetric substrate. Both granzymes were assayed against different concentrations of substrate and initial rate determined and plotted against the substrate concentration. Data represent mean \pm S.D. of three independent experiments. (h) Both wtGB and KR243/244AA GB cleaved to the same extent NDUFS1-D255A from 721.221 cell lysate. Blot is representative of three independent experiments. (i) GB expression in parental YT-Indy, YT-Indy silenced for GB, GM and GK (YT-shGzm), and YT-shGzm where wtGB or KR243/244AA GB were reintroduced (YT-wtGB and YT-KR/AA GB, respectively). Blot is representative of three independent experiments. (j) GB is delivered to a similar extent into target HeLa-CD80 cells by YT-wtGB and YT-KR/AA GB. Effector/target conjugates were formed for 30 min and GB (green) was stained by immunofluorescence. (k) The 721.221 target cells were incubated with YT-wtGB or YT-KR/AA GB and PARP-1 and Bid cleavage monitored by Western blot. $\kappa$-Light chain was used as a loading control specific to 721-221. YT-wtGB and YT-KR/AA GB were used to attack HeLa-CD80 cells expressing either cytosolic (I) or mitochondrial (m) GB-FRET reporter. Data represent mean \pm S.E.M. of three independent experiments. (n) CFSE-labeled HeLa-CD80 target cells were incubated for $2 \mathrm{~h}$ with YT-wtGB (green bars) or YT-KR/AA GB (red bars). ROS production was measured by dihydroethidium (DHE) staining and flow cytometry analysis gating on the CFSE-positive cells. (o) Parental YT-Indy, YT-shGzm, YT-wtGB and YT-KR/AA GB were used to kill HeLa-CD80 $0^{+}$cells using calcein-AM release assay. Data represent mean \pm S.D. of three independent experiments. ${ }^{*} P \leq 0.05,{ }^{* *} P \leq 0.01$ and ${ }^{* *} P \leq 0.001$ (two-sided $t$-test). (p) A total of $2 \times 10^{6}$ U251 glioma cells expressing Renilla luciferase were implanted subcutaneously in nonobese diabetic/severe combined immunodeficiency (NOD/SCID) mice. Tumor-bearing mice were left untreated (control) or adoptively transferred with $10^{7} \mathrm{YT}$-shGzm, YT-wtGB or YT-KR/AA GB at day 8 post-tumor implantation. Tumor growth was followed by luminometry at day 3, 10 and 17 post-tumor implantation. (q) Same as in (p), quantification of the bioluminescence imaging (BLI) signal, $n=5$ mice per group. ${ }^{*} P \leq 0.05$ 
a

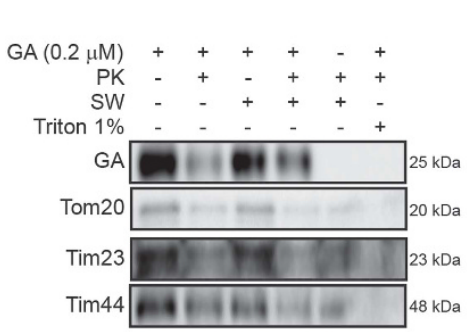

b

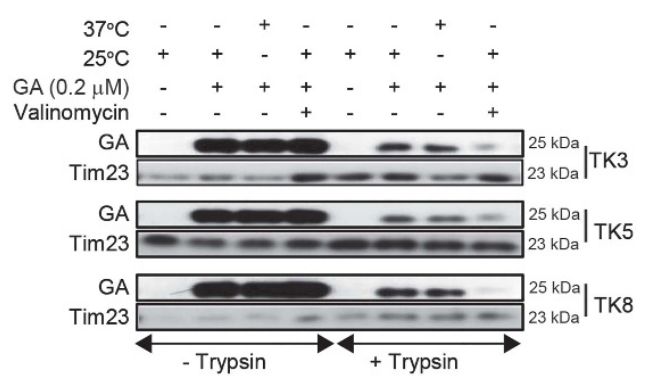

C

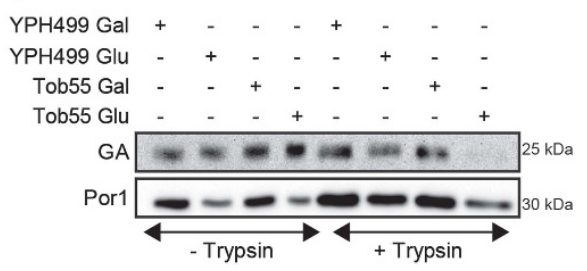

d
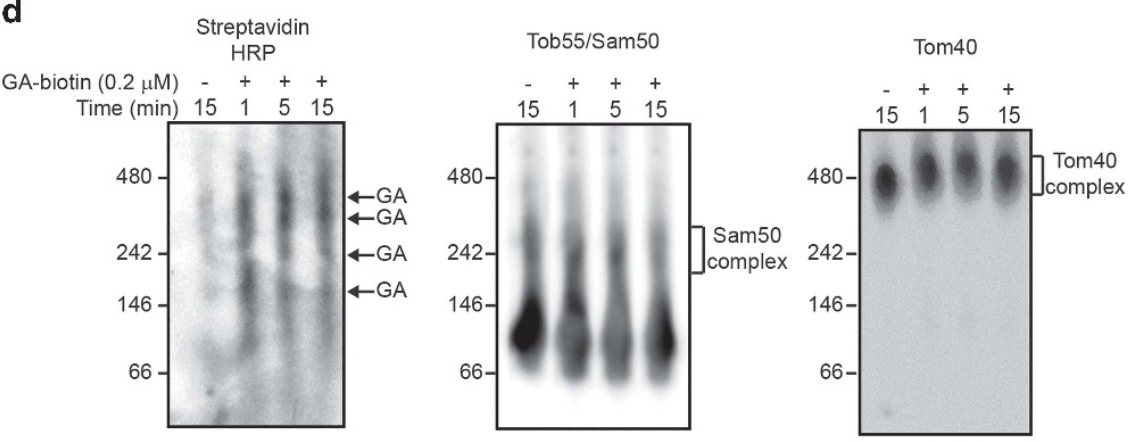

e
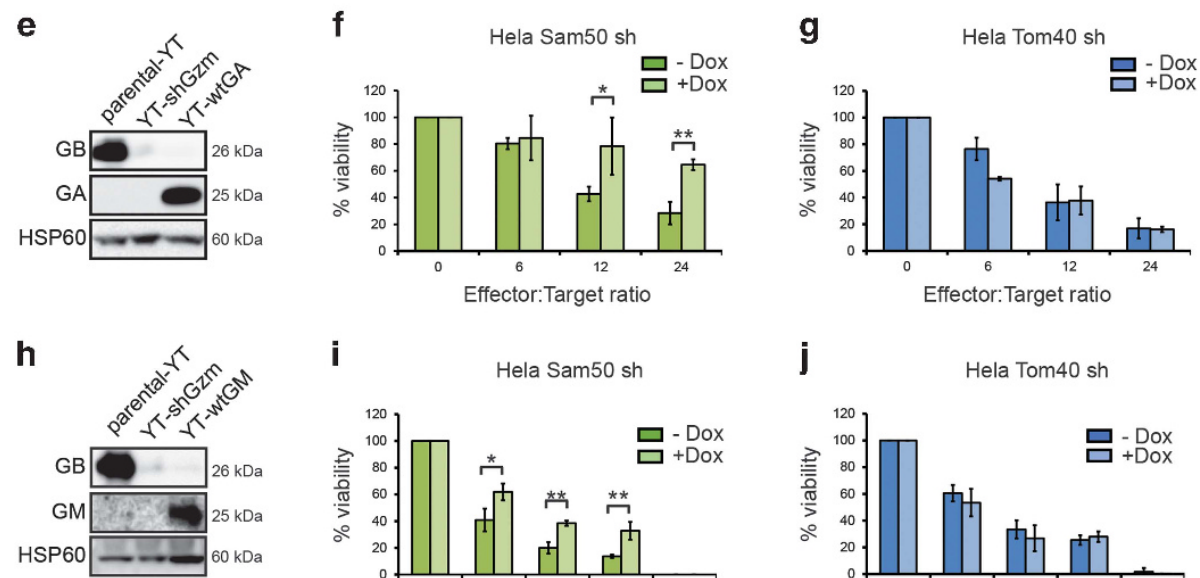

i

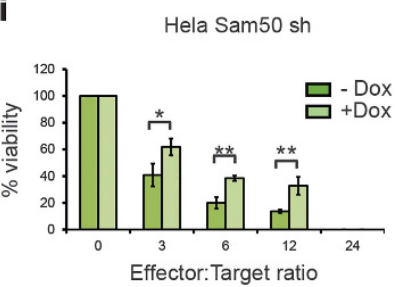

j

Hela Tom40 sh

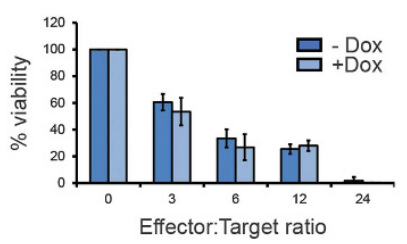

Figure 5 Tob55/Sam50-deficient cells are resistant to GA and GM-induced cell death. (a) Freshly purified yeast mitochondria were incubated or not with recombinant GA in import buffer before gentle treatment with proteinase $\mathrm{K}(\mathrm{PK})$ in the absence or presence of a swelling step or direct solubilization with $1 \%$ Triton X-100. (b) Mitochondria purified from TK8, TK3 and TK5 yeast strain were used in import assay for wild-type (wt) GA at $25^{\circ} \mathrm{C}$ or $37^{\circ} \mathrm{C}$. (c) Mitochondria purified from parental or gal-Tob55-His yeast strains grown in galactose or glucose were used in an import assay for GA. (d) Mitochondria purified from Tob55 yeast strain grown in galactose medium were used in import assay for biotinylated GA (GA-biotin). Samples were resolved by Blue-Native gel electrophoresis and analyzed by western blot with streptavidin-HRP, anti-yeast Tob55 or anti-yeast Tom40. (e) Expression of GA in parental YT-Indy, YT-shGzm and YT-shGzm transduced with lentiviral construct for GA (YT-wtGA). HeLa silenced for Tob55/Sam50 (f) or Tom40 (g) following DOX treatment are used as targets for YT-wtGA. (h) Expression of GM in parental YT-Indy, YT-shGzm and YT-shGzm transduced with lentiviral construct for GM (YT-wtGM). (i and j) Same as in (f and $\mathbf{g}$ ) using YT-Indy killer cells expressing GM. Western blots are representative of three independent experiments and histograms are mean \pm S.D. of three independent experiments. ${ }^{*} P \leq 0.05,{ }^{*} P \leq 0.01$ (two-sided $t$-test)

SSC $1-3^{56}$ were defective in their ability to import both GA and $\mathrm{GB}$, suggesting that the $\mathrm{mH} H \mathrm{sp} 70$ is necessary for GA and GB to cross the inner membrane (Figures $7 e$ and f).

Altogether, our results indicate that Tob55/Sam50 is required for GB, GA and caspase- 3 to cross the outer mitochondrial membrane, whereas Tim22 and $\mathrm{mtHsp} 70$ are necessary for $G A$ and $G B$ to cross the inner mitochondrial membrane.

\section{Discussion}

We have found that GB enters the yeast and mouse mitochondria through a conserved noncanonical pathwaydependent on Tob55/Sam50, Tim22 and mtHsp70. This mitochondrial entry did not require GB catalytic activity, but instead was dependent on its $\mathrm{C}$-terminal residues Lys and Arg 243-244. Mutant GB that cannot enter the mitochondria is less potent at inducing ROS and cell death. Reciprocally, cells with 
a

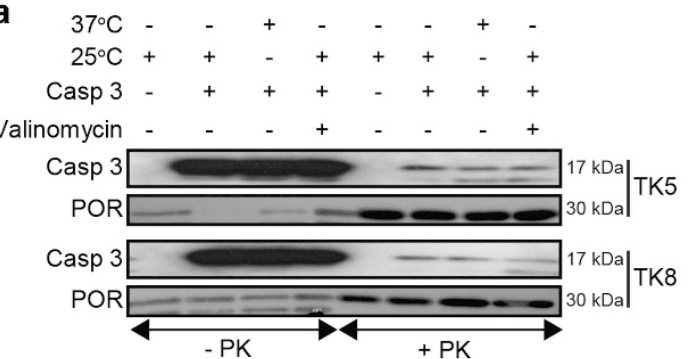

b

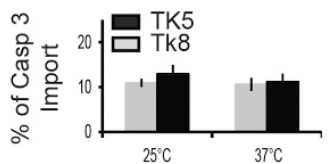

C

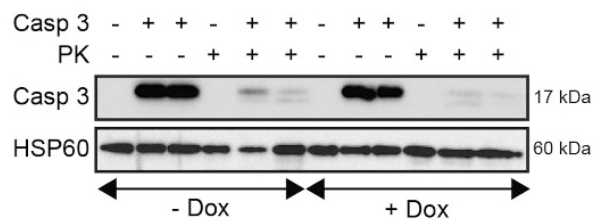

d

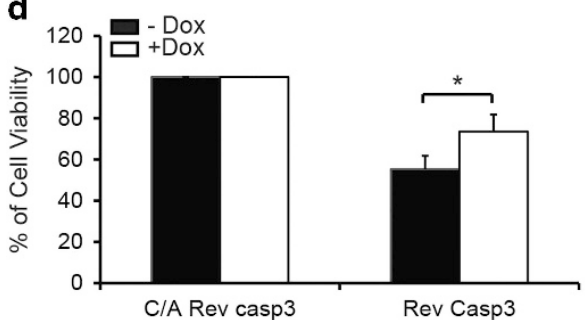

Figure 6 Caspase-3 enters the mitochondria in a Tob55/Sam50-dependent manner. (a) Mitochondria purified from TK8 or TK5 yeast strains were used for import of active recombinant human caspase-3. (b) Same as in (a) mean \pm S.D. of three independent experiments. (c) Mitochondria purified from HeLa cells expressing DOX-inducible short hairpin RNA (shRNA) targeting Sam50 grown for 3 days in the absence or presence of DOX were used in import assay for recombinant active human caspase-3. Western blots are all representative of three independent experiments. (d) HeLa cells silenced for Tob55/Sam50 for 4 days total. The cells were transfected on day 3 of DOX induction with construct encoding inactive C/A Rev-caspase-3 or active Rev-caspase-3. Cell viability was monitored $24 \mathrm{~h}$ post-transfection by methylene blue staining

Sam50 knockdown become resistant to GB, GA, GM and active caspase-3-mediated cell death.

GB is a fully mature protein that does not have a mitochondrial matrix-targeting signal. In fact, following its final proteolytic activation in the cytotoxic granules, GB hydrophobic N-terminal end is buried in a groove surrounded on each side by loops Trp141-Ser152 and Gly184-Gly196 and at the bottom by $\beta$-strands GIn135-Gly140 and His153-Val162. ${ }^{38}$ On the other side, the polar GB C-terminal tail from the $a$-helix Val235-Thr241 to the tetrapeptide Met242-Tyr245 is accessible $^{38}$ and harbors the targeting signal, which is not recognizable as an amphipathic helix and is not cleavable. Residues Lys and Arg 243-244 are necessary for this function. In contrast, His236 and Lys 239-240, other cationic residues important for perforin-mediated cytosolic delivery of GB in target cells, ${ }^{48}$ are not essential for the mitochondrial entry. Substitution of Lys and Arg 243-244 did not impair GB catalytic activity, in agreement with their position at the tip of the flexible C-terminal tail. It can be noted from the report of Trapani's group ${ }^{48}$ that similar substitutions on the GB CS2 cationic site did not alter its catalytic activity while reducing the accumulation of the mutated GB in intracellular compartment leading to fold loss in cytotoxicity. Although mutant YT-KR/AA GB is not efficiently delivered to target cells by perforin in loading experiment, it is efficiently delivered to target cells during killer cell attack. It was recently shown that effector cells exert synaptic force to enhance perforin activity, ${ }^{57}$ explaining the discrepancy between the loading experiment and the physiological effector/target interaction. However, GB $\mathrm{KR} / \mathrm{AA}$, which cannot enter the mitochondria is significantly impaired in its ability to trigger ROS and cell death both in vitro and in vivo, indicating that GB mitochondrial entry is necessary for efficient cell death induction in agreement with Jacquemin et al. ${ }^{18}$
Tob55/Sam50 belongs to the bacterial $\beta$-barrel-shaped channels of the Omp85 family. ${ }^{58,59}$ The primary function of this protein family is to integrate $\beta$-barrel proteins into the outer membranes of bacteria or mitochondria. However, Toc75, the central import pore of the translocase of the outer membrane of chloroplasts, also belongs to the Omp85 family, implying that proteins structurally similar to Tob55/Sam50 can function as transport pores. ${ }^{60}$ Tob55/Sam50 channel is $\sim 7-8 \mathrm{~nm}$ large enough to accommodate the 16-22 $\beta$-strands of a $\beta$-barrel protein. ${ }^{61}$ It is even speculated to work as an oligomer to facilitate the lateral release of the $\beta$-barrel protein being membrane-inserted, which in this context could increase its channel cutoff. ${ }^{62,63}$ However, even as a monomer this channel is large enough to accommodate the $3.5 \times 5 \mathrm{~nm}$ diameter of the GB ellipsoid. ${ }^{38}$ Interestingly, in agreement with our findings, it was previously reported that Tob55/Sam50 channel could have a role in apoptosis. ${ }^{61,64} \mathrm{Tim} 22$ and $\mathrm{mtH}$ sp70 are required for granzyme to cross the inner membrane. Tim22 is the core channel of the metabolite carrier translocase complex. ${ }^{65-68}$ Tim22 has a dynamic pore size ranging from 1.1 to $1.8 \mathrm{~nm}$, which is still below the size of granzyme. How Tim22 releases the cargo protein into the lipid bilayer is still unknown, but likely involves conformational changes that could be exploited by hijacker proteins such as granzymes. The requirement for $\mathrm{mtH}$ sp70 in granzyme mitochondrial import is less surprising as GA and GB have been shown to interact strongly with cytosolic Hsp27, Hsp70 and Hsp90. ${ }^{69-71}$ SSC1-3 allele presents a G56S mutation in the mtHsp70 ATPase domain, ${ }^{72}$ indicating that GB might cross the inner mitochondrial membrane in a forceful manner. Moreover SSC1-2 yeast strain carries an allele of $\mathrm{mtHsp} 70$ that poorly interact with Tim44 at $37^{\circ} \mathrm{C} .{ }^{73}$ Yet, SSC1-2 mitochondria imported very well $\mathrm{GB}$ and $\mathrm{GA}$ at both 25 and $37^{\circ} \mathrm{C}$, indicating that it is most likely a presequence translocase-associated motor (PAM) 
a

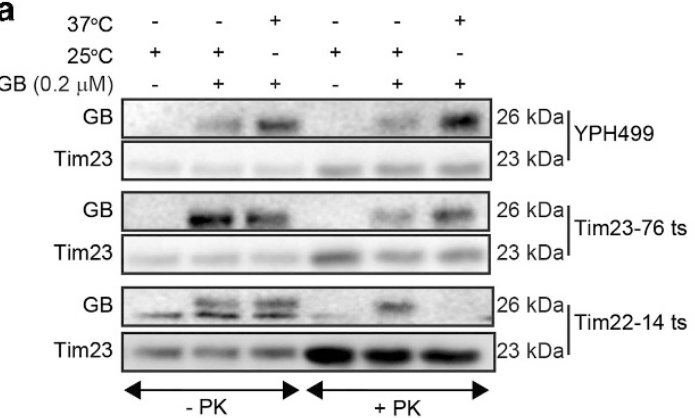

C
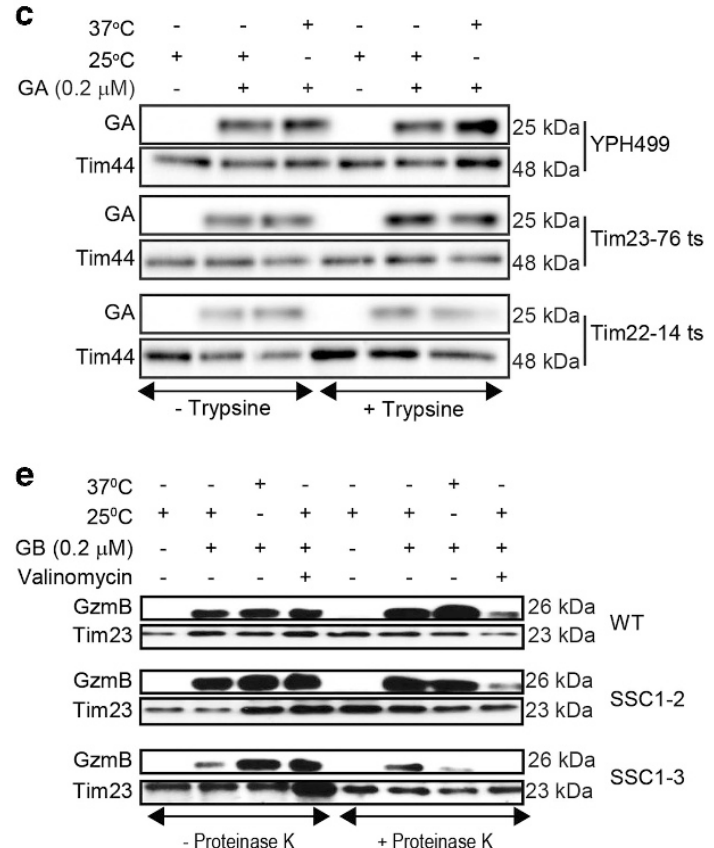

b

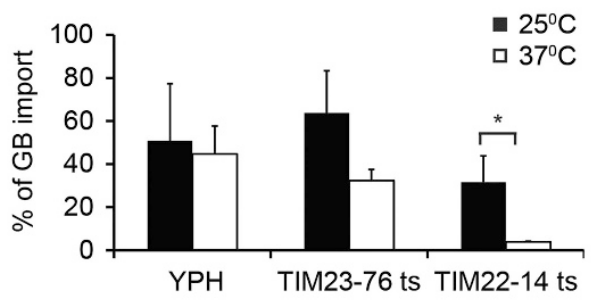

d
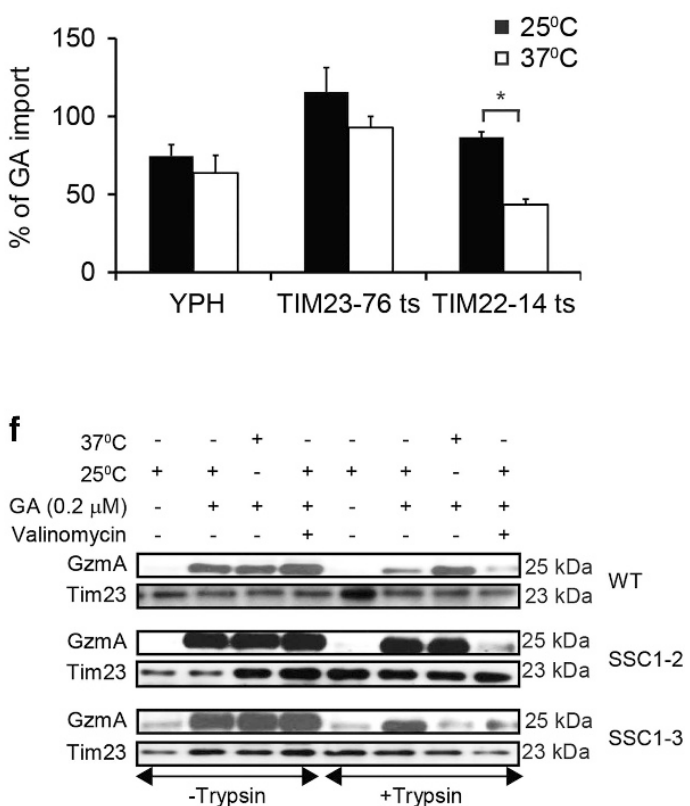

Figure 7 GB crosses the inner mitochondrial membrane in a Tim22 channel-dependent manner. (a) Mitochondria purified from parental yeast strain YPH499 or YPH499 strain carrying thermosensitive alleles of Tim23 channel (Tim23-76ts) or Tim22 (Tim22-14ts) were used in import assay of GB at $25^{\circ} \mathrm{C}$ or $37^{\circ} \mathrm{C}$ permissive and non-permissive temperature, respectively. Western blots are representative of three independent experiments. (b) Same as in (a) mean \pm .S.E.M. of three independent experiments. ${ }^{*} P \leq 0.05$, two-sided $t$-test). (c and $\mathbf{d}$ ) Same as in (a and b) using GA. Mitochondria purified from parental yeast strain YPH499 or YPH499 strain carrying thermosensitive alleles of mtHsp70 SSC1-2 unable to bind the cargo or SSC1-3 unable to pull the cargo were used in import assay of GB (e) or GA (f) at $25^{\circ} \mathrm{C}$ or $37^{\circ} \mathrm{C}$ permissive and non-permissive temperature, respectively

complex-independent process. Additionally, we also reported that GB disrupts the mitochondrial cristae junction, possibly contributing to GB forceful passage across the inner membrane. ${ }^{18}$ Additional experiments are needed to test this possibility.

Interestingly, silencing Tob55/Sam50 but not Tom40 also rendered cells resistant to killer cells expressing either GA or $\mathrm{GM}$, in agreement with the ability of all five human granzymes to colocalize with the mitochondria. Because human and mouse granzyme share the same structure, ${ }^{47}$ it is likely they follow this same pathway into the mitochondria. Interestingly, we also noted that active caspase- 3 uses a similar route to the mitochondria.

Conceptually, thinking at the TOM-TIM23 complexes not only as translocases but also as safe-keepers of the mitochondrial integrity through their high selectivity, the fact that cytotoxic molecules aimed at destroying the mitochondria use Tob55/Sam50-Tim22 as a side doors to access the mitochondria makes sense. Both Tob55/Sam50 and Tim22 are dedicated to the insertion of proteins in the mitochondrial membrane and were not intended to be used as translocases. It is therefore possible that some mechanistic aspect of this common function could be used unconventionally by the cytotoxic molecules. In this regard, granzyme mitochondrial entry breaks all the codes of mitochondrial import, something that could be expected from proteins aimed at destroying the mitochondrial functions for irrevocable cell death. Finally, preventing GB, GA, GM and caspase-3 cytotoxic factors to access the mitochondria compromise their ability to induce cell death, suggesting that this is probably an unanticipated critical step for apoptosis. In conclusion, we have demonstrated that cytotoxic molecules gain access to the mitochondria in a Tob55/Sam50-, Tim22- and mtHsp70-dependent manner to induce cell death. 


\section{Conflict of Interest}

The authors declare no conflict of interest.

Acknowledgements. This work was supported by ERC starting Grant ERC-2010-StG_20091118, Ambizione SNSF PZO0P3 126710/1 and subsidies from the Carlos and Elsie de Reuter Foundation and Schmidheiny Foundation and Boninchi Foundation. We thank Professor Doron Rapaport, Professor Marco Colombini, Dr Dejana Mokranjac, Professor Nikolaus Pfanner, Dr Bernard Guiard, Professor Elizabeth A. Craig and Dr Ines Mendes Pinto for yeast strains and antibodies. We also thank Olivier Dupont and Christian Vesin for technical assistance.

1. Chowdhury D, Lieberman J. Death by a thousand cuts: granzyme pathways of programmed cell death. Annu Rev Immunol 2008; 26: 389-420.

2. Law RH, Lukoyanova N, Voskoboinik I, Caradoc-Davies TT, Baran K, Dunstone MA et al. The structural basis for membrane binding and pore formation by lymphocyte perforin. Nature 2010; 468: 447-451

3. Thiery J, Keefe D, Boulant S, Boucrot E, Walch M, Martinvalet D et al. Perforin pores in the endosomal membrane trigger the release of endocytosed granzyme $B$ into the cytosol of target cells. Nat Immunol 2011; 12: 770-777.

4. Walch M, Dotiwala F, Mulik S, Thiery J, Kirchhausen T, Clayberger $C$ et al. Cytotoxic cells kill intracellular bacteria through granulysin-mediated delivery of granzymes. Cell 2014; 157: 1309-1323.

5. Andrade F, Roy S, Nicholson D, Thornberry N, Rosen A, Casciola-Rosen L. Granzyme B directly and efficiently cleaves several downstream caspase substrates: implications for CTL-induced apoptosis. Immunity 1998; 8: 451-460.

6. Cullen SP, Adrain C, Luthi AU, Duriez PJ, Martin SJ. Human and murine granzyme B exhibit divergent substrate preferences. J Cell Biol 2007; 176: 435-444.

7. Darmon AJ, Nicholson DW, Bleackley RC. Activation of the apoptotic protease CPP32 by cytotoxic T-cell-derived granzyme B. Nature 1995; 377: 446-448.

8. Goping IS, Barry M, Liston P, Sawchuk T, Constantinescu G, Michalak KM et al. Granzyme B-induced apoptosis requires both direct caspase activation and relief of caspase inhibition. Immunity 2003; 18: 355-365.

9. Heibein JA, Barry M, Motyka B, Bleackley RC. Granzyme B-induced loss of mitochondrial inner membrane potential (Delta Psi $\mathrm{m}$ ) and cytochrome $\mathrm{c}$ release are caspase independent. J Immunol 1999; 163: 4683-4693.

10. Sutton VR, Davis JE, Cancilla M, Johnstone RW, Ruefli AA, Sedelies $K$ et al. Initiation of apoptosis by granzyme $B$ requires direct cleavage of bid, but not direct granzyme B-mediated caspase activation. J Exp Med 2000; 192: 1403-1414.

11. Alimonti JB, Shi L, Baijal PK, Greenberg AH. Granzyme B induces BID-mediated cytochrome $c$ release and mitochondrial permeability transition. J Biol Chem 2001; 276: 6974-6982.

12. Casciola-Rosen L, Garcia-Calvo M, Bull HG, Becker JW, Hines T, Thornberry NA et al. Mouse and human granzyme $B$ have distinct tetrapeptide specificities and abilities to recruit the bid pathway. J Biol Chem 2007; 282: 4545-4552.

13. Han J, Goldstein LA, Hou W, Froelich CJ, Watkins SC, Rabinowich H. Deregulation of mitochondrial membrane potential by mitochondrial insertion of granzyme $\mathrm{B}$ and direct $\mathrm{Hax}-$ 1 cleavage. J Biol Chem 2010; 285: 22461-22472.

14. Heusel JW, Wesselschmidt RL, Shresta S, Russell JH, Ley TJ. Cytotoxic lymphocytes require granzyme $B$ for the rapid induction of DNA fragmentation and apoptosis in allogeneic target cells. Cell 1994; 76: 977-987.

15. MacDonald G, Shi L, Vande Velde C, Lieberman J, Greenberg AH. Mitochondria-dependent and -independent regulation of Granzyme B-induced apoptosis. J Exp Med 1999; 189: 131-144.

16. Wang GQ, Wieckowski E, Goldstein LA, Gastman BR, Rabinovitz A, Gambotto A et al. Resistance to granzyme B-mediated cytochrome $c$ release in Bak-deficient cells. J Exp Med 2001; 194: 1325-1337.

17. Waterhouse NJ, Sedelies KA, Browne KA, Wowk ME, Newbold A, Sutton VR et al. A central role for Bid in granzyme B-induced apoptosis. J Biol Chem 2005; 280: 4476-4482.

18. Jacquemin G, Margiotta D, Kasahara A, Bassoy EY, Walch M, Thiery J et al. Granzyme B-induced mitochondrial ROS are required for apoptosis. Cell Death Differ 2015; 22: 862-874.

19. Bauer MF, Sirrenberg C, Neupert W, Brunner M. Role of Tim23 as voltage sensor and presequence receptor in protein import into mitochondria. Cell 1996; 87: 33-41.

20. Hill K, Model K, Ryan MT, Dietmeier K, Martin F, Wagner R et al. Tom40 forms the hydrophilic channel of the mitochondrial import pore for preproteins [see comment]. Nature 1998; 395: 516-521.

21. Malhotra K, Sathappa M, Landin JS, Johnson AE, Alder NN. Structural changes in the mitochondrial Tim23 channel are coupled to the proton-motive force. Nat Struct Mol Biol 2013; 20: 965-972.

22. Mokranjac D, Neupert W. The many faces of the mitochondrial TIM23 complex. Biochim Biophys Acta 2010; 1797: 1045-1054

23. Mokranjac D, Paschen SA, Kozany C, Prokisch H, Hoppins SC, Nargang FE et al. Tim50, a novel component of the TIM23 preprotein translocase of mitochondria. EMBO J 2003a; 22: 816-825.
24. Mokranjac D, Sichting M, Neupert W, Hell K. Tim14, a novel key component of the import motor of the TIM23 protein translocase of mitochondria. EMBO J 2003b; 22: 4945-4956.

25. Neupert W. A perspective on transport of proteins into mitochondria: a myriad of open questions. J Mol Biol 2015; 427: 1135-1158.

26. Popov-Celeketic D, Mapa K, Neupert W, Mokranjac D. Active remodelling of the TIM23 complex during translocation of preproteins into mitochondria. EMBO J 2008; 27: $1469-1480$

27. Wallace DC. Mitochondrial diseases in man and mouse. Science 1999; 283: 1482-1488.

28. Young JC, Hoogenraad NJ, Hartl FU. Molecular chaperones Hsp90 and Hsp70 deliver preproteins to the mitochondrial import receptor Tom70. Cell 2003; 112: 41-50.

29. Choi PJ, Mitchison TJ. Imaging burst kinetics and spatial coordination during serial killing by single natural killer cells. Proc Natl Acad Sci USA 2013; 110: 6488-6493.

30. Kozjak-Pavlovic V, Ott C, Gotz M, Rudel T. Neisserial Omp85 protein is selectively recognized and assembled into functional complexes in the outer membrane of human mitochondria. J Biol Chem 2011; 286: 27019-27026.

31. Kozjak-Pavlovic V, Prell F, Thiede B, Gotz M, Wosiek D, Ott C et al. C1orf163/RESA1 is a novel mitochondrial intermembrane space protein connected to respiratory chain assembly. J Mol Biol 2014; 426: 908-920.

32. Haucke V, Lithgow T, Rospert S, Hahne K, Schatz G. The yeast mitochondrial protein import receptor Mas20p binds precursor proteins through electrostatic interaction with the positively charged presequence. J Biol Chem 1995; 270: 5565-5570.

33. Krimmer T, Rapaport D, Ryan MT, Meisinger C, Kassenbrock CK, Blachly-Dyson E et al. Biogenesis of porin of the outer mitochondrial membrane involves an import pathway via receptors and the general import pore of the TOM complex. J Cell Biol 2001; 152: 289-300.

34. Rojo EE, Stuart RA, Neupert W. Conservative sorting of F0-ATPase subunit 9: export from matrix requires delta $\mathrm{pH}$ across inner membrane and matrix ATP. EMBO J 1995; 14 : 3445-3451.

35. Schneider HC, Berthold J, Bauer MF, Dietmeier K, Guiard B, Brunner M et al.. Mitochondrial Hsp70/MIM44 complex facilitates protein import. Nature 1994; 371: 768-774.

36. Lithgow T, Junne T, Wachter C, Schatz G. Yeast mitochondria lacking the two import receptors Mas20p and Mas70p can efficiently and specifically import precursor proteins. $J$ Biol Chem 1994b; 269: 15325-15330.

37. Lithgow T, Junne T, Suda K, Gratzer S, Schatz G. The mitochondrial outer membrane protein Mas22p is essential for protein import and viability of yeast. Proc Natl Acad Sci USA 1994a; 91: 11973-11977.

38. Estebanez-Perpina E, Fuentes-Prior P, Belorgey D, Braun M, Kiefersauer R, Maskos K et al. Crystal structure of the caspase activator human granzyme $B$, a proteinase highly specific for an Asp-P1 residue. Biol Chem 2000; 381: 1203-1214.

39. Lee AC, Xu X, Blachly-Dyson E, Forte M, Colombini M. The role of yeast VDAC genes on the permeability of the mitochondrial outer membrane. J Membr Biol 1998; 161: 173-181.

40. Stein CA, Colombini M. Specific VDAC inhibitors: phosphorothioate oligonucleotides. $J$ Bioenerg Biomembr 2008; 40: 157-162.

41. Hiller S, Abramson J, Mannella C, Wagner G, Zeth K. The 3D structures of VDAC represent a native conformation. Trends Biochem Sci 2010; 35: 514-521.

42. Zeth K. Structure and evolution of mitochondrial outer membrane proteins of beta-barre topology. Biochim Biophys Acta 2010; 1797: 1292-1299.

43. Darshi M, Mendiola VL, Mackey MR, Murphy AN, Koller A, Perkins GA et al. ChChd3, an inner mitochondrial membrane protein, is essential for maintaining crista integrity and mitochondrial function. J Biol Chem 2011; 286: 2918-2932.

44. Darshi M, Trinh KN, Murphy AN, Taylor SS. Targeting and import mechanism of coiled-coil helix coiled-coil helix domain-containing protein 3 (ChChd3) into the mitochondrial intermembrane space. J Biol Chem 2012; 287: 39480-39491.

45. Walther DM, Papic D, Bos MP, Tommassen J, Rapaport D. Signals in bacterial beta-barre proteins are functional in eukaryotic cells for targeting to and assembly in mitochondria. Proc Natl Acad Sci USA 2009; 106: 2531-2536.

46. Chan NC, Lithgow T. The peripheral membrane subunits of the SAM complex function codependently in mitochondrial outer membrane biogenesis. Mol Biol Cell 2008; 19 : 126-136.

47. Sattar R, Ali SA, Abbasi A. Bioinformatics of granzymes: sequence comparison and structural studies on granzyme family by homology modeling. Biochem Biophys Res Commun 2003; 308: 726-735.

48. Bird CH, Sun J, Ung K, Karambalis D, Whisstock JC, Trapani JA et al. Cationic sites on granzyme $\mathrm{B}$ contribute to cytotoxicity by promoting its uptake into target cells. $\mathrm{Mol} \mathrm{Cell} \mathrm{Biol}$ 2005; 25: 7854-7867.

49. Ott C, Dorsch E, Fraunholz M, Straub S, Kozjak-Pavlovic V. Detailed analysis of the human mitochondrial contact site complex indicate a hierarchy of subunits. PLOS ONE 2015; 10: e0120213.

50. Chacinska A, Koehler CM, Milenkovic D, Lithgow T, Pfanner N. Importing mitochondrial proteins: machineries and mechanisms. Cell 2009; 138: 628-644.

51. Petrosillo G, Ruggiero FM, Paradies G. Role of reactive oxygen species and cardiolipin in the release of cytochrome c from mitochondria. FASEB J 2003; 17: 2202-2208.

52. Srinivasula SM, Ahmad M, MacFarlane M, Luo Z, Huang Z, Fernandes-Alnemri T et al. Generation of constitutively active recombinant caspases -3 and -6 by rearrangement of their subunits. J Biol Chem 1998; 273: 10107-10111.

53. Ricci JE, Munoz-Pinedo C, Fitzgerald P, Bailly-Maitre B, Perkins GA, Yadava N et al. Disruption of mitochondrial function during apoptosis is mediated by caspase cleavage of the p75 subunit of complex I of the electron transport chain. Cell 2004; 117: 773-786. 
54. van der Laan M, Meinecke M, Dudek J, Hutu DP, Lind M, Perschil I et al. Motor-free mitochondrial presequence translocase drives membrane integration of preproteins. Nat Cell Biol 2007; 9: 1152-1159.

55. Wagner K, Gebert N, Guiard B, Brandner K, Truscott KN, Wiedemann N et al. The assembly pathway of the mitochondrial carrier translocase involves four preprotein translocases. Mol Cell Biol 2008; 28: 4251-4260.

56. Gambill BD, Voos W, Kang PJ, Miao B, Langer T, Craig EA et al. A dual role for mitochondria heat shock protein 70 in membrane translocation of preproteins. J Cell Biol 1993; 123 109-117.

57. Basu R, Whitlock BM, Husson J, Le Floc'h A, Jin W, Oyler-Yaniv A et al. Cytotoxic T cells use mechanical force to potentiate target cell killing. Cell 2016; 165: 100-110.

58. Gentle I, Gabriel K, Beech P, Waller R, Lithgow T. The Omp85 family of proteins is essential for outer membrane biogenesis in mitochondria and bacteria. J Cell Biol 2004; 164: $19-24$.

59. Moslavac S, Mirus O, Bredemeier R, Soll J, von Haeseler A, Schleiff E. Conserved pore-forming regions in polypeptide-transporting proteins. FEBS J 2005; 272: 1367-1378.

60. Andres C, Agne B, Kessler F. The TOC complex: preprotein gateway to the chloroplast. Biochim Biophys Acta 2010; 1803: 715-723.

61. Paschen SA, Waizenegger $T$, Stan $T$, Preuss $M$, Cyrklaff $M$, Hell $K$ et al. Evolutionary conservation of biogenesis of beta-barrel membrane proteins. Nature 2003; 426: 862-866.

62. Klein A, Israel L, Lackey SW, Nargang FE, Imhof A, Baumeister W et al. Characterization of the insertase for beta-barrel proteins of the outer mitochondrial membrane. J Cell Biol 2012; 199: 599-611.

63. Kutik S, Stojanovski D, Becker L, Becker T, Meinecke M, Kruger V et al. Dissecting membrane insertion of mitochondrial beta-barrel proteins. Cell 2008; 132: 1011-1024.
64. Pavlov EV, Priault M, Pietkiewicz D, Cheng EH, Antonsson B, Manon S et al. A novel, high conductance channel of mitochondria linked to apoptosis in mammalian cells and Bax expression in yeast. J Cell Biol 2001; 155: 725-731.

65. Gebert N, Gebert M, Oeljeklaus S, von der Malsburg K, Stroud DA, Kulawiak B et al. Dual function of Sdh3 in the respiratory chain and TIM22 protein translocase of the mitochondrial inner membrane. Mol Cell 2011; 44: 811-818.

66. Kerscher O, Sepuri NB, Jensen RE. Tim18p is a new component of the Tim54p-Tim22p translocon in the mitochondrial inner membrane. Mol Biol Cell 2000; 11: 103-116.

67. Kovermann P, Truscott KN, Guiard B, Rehling P, Sepuri NB, Muller H et al. Tim22, the essential core of the mitochondrial protein insertion complex, forms a voltage-activated and signal-gated channel. Mol Cell 2002; 9: 363-373.

68. Rehling P, Model K, Brandner K, Kovermann P, Sickmann A, Meyer HE et al. Protein insertion into the mitochondrial inner membrane by a twin-pore translocase. Science 2003; 299: 1747-1751.

69. Beresford PJ, Zhang D, Oh DY, Fan Z, Greer EL, Russo ML et al. Granzyme A activates an endoplasmic reticulum-associated caspase-independent nuclease to induce single-stranded DNA nicks. J Biol Chem 2001; 276: 43285-43293.

70. Martinvalet D, Dykxhoorn DM, Ferrini R, Lieberman J. Granzyme A cleaves a mitochondrial complex I protein to initiate caspase-independent cell death. Cell 2008; 133: 681-692.

71. Osterloh $A$, Breloer M. Heat shock proteins: linking danger and pathogen recognition. Med Microbiol Immunol 2008; 197: 1-8.

72. Krayl M, Lim JH, Martin F, Guiard B, Voos W. A cooperative action of the ATP-dependent import motor complex and the inner membrane potential drives mitochondrial preprotein import. Mol Cell Biol 2007; 27: 411-425.

73. D'Silva $\mathrm{P}$, Liu Q, Walter W, Craig EA. Regulated interactions of mtHsp70 with Tim44 at the translocon in the mitochondrial inner membrane. Nat Struct Mol Biol 2004; 11: 1084-1091.

Supplementary Information accompanies this paper on Cell Death and Differentiation website (http://www.nature.com/cdd) 